Joseph Y. Ting

\section{Research accomplishments that are too good to be true: comment}

Accepted: 26 December 2013

Published online: 30 January 2014

(C) Springer-Verlag Berlin Heidelberg and ESICM 2014

A reply to this comment is available at doi:10.1007/s00134-014-3220-0.

Dear Editor,

In his real-life vignette series

"Research accomplishments that are too good to be true," Ioannidis [1] discusses useful strategies that hold potential to reduce biased or misleading reporting and deliberate clinical research misconduct. It is reasonable to surmise that the publish-or-perish instinct to achieve academic advancement can override personal ethics and the moral selfregulation that proscribes clinical research transparency and credibility. In this setting, tightening external oversight and imposing disciplinary sanctions could deter deliberate exaggeration and fabrication of research findings [2].

I would add surveillance of a clinical trial whilst it is being conducted, the imposition of conflict of interest declaration as a pre-requisite to journal publication and robust feedback mechanisms that allow post-publication scrutiny of controversial findings to Ioannidis' recommendations. Selective outcome reporting leading to biased treatment and adverse effect estimates could be discerned by independent re-evaluation of full outcome data that are made publicly available after publication of a clinical trial $[1,3]$. However, empowering existing data and safety committees to monitor for selective data collection and use while the study is in progress improves the validity and credibility of submitted manuscripts, eliminates the need for resource-intensive postpublication re-evaluation, and averts disseminating biased estimates to journal audiences in the first place [4]

Conflict of interest is complex, existing at multiple levels-from that of the individual, the research team, the institution in which the work is done and beyond, to the equitable allocation of health research funding. Although its declaration is advocated to discern pharmaceutical sponsorship that biases treatment recommendations, subtle conflict of interest may not be evident to even the most impartial researcher at the time of manuscript submission. Furthermore, there is limited evidence that overt declaration of conflict of interest negatively affects reader perception in terms of interest, importance, relevance and believability of a published study [5].

Journals could adopt policies that mandate reader concerns to be satisfactorily addressed by study authors and journal editors, with ongoing disagreements resolved by independent arbitration. Post-publication debate is fostered by reader scepticism, contrarian opinion and author counter-responses being housed within the forum of a journal's "Letters to the Editor" or end-ofarticle comments section. What could be done better is for all reasonably argued responses to be automatically published and appended to the original study online, in order to maintain balance and avoid accusations of editorial bias. For instance, "Rapid Responses" has been a feedback feature at the British Medical Journal for several years.

The issue, however, should be deemed to go beyond research misconduct to the material risk of harming patients. If there is no internal dissent and a published study had been accepted at face value without further scrutiny, a supposedly effective treatment could have been recommended to patients as a primary course of action and for those who fail conventional treatment, or be wastefully investigated further in a large definitive clinical trial. Patients are exposed to a speculative if not detrimental intervention by being given false hope, receiving non-beneficial treatment and forgoing therapies that do work, as well as suffering unnecessary side effects.

Conflicts of interest On behalf of all authors, the corresponding author states that there is no conflict of interest.

\section{References}

1. Ioannidis JPA (2014) Research accomplishments that are too good to be true. Intensive Care Med 40:99-101. doi: 10.1007/s00134-013-3100-Z

2. Zinner DE, DesRoches CM, Bristol SJ, Clarridge B, Campbell EG (2010) Tightening conflict-of-interest policies: the impact of 2005 ethics rules at the NIH. Acad Med 85:1685-1691

3. Chan A-W (2011) Access to clinical trial data. BMJ 342:d80

4. Ting JY (2011) Access to clinical trial data. Data monitoring committees and selective outcome reporting. BMJ 342:d635

5. Chaudhry S, Schroter S, Smith R, Morris J (2002) Does declaration of competing interests affect readers' perceptions? A randomised trial. BMJ 325:1391-1392

\section{J. Y. Ting (-)}

School of Public Health and Social Work, Queensland University of Technology,

Brisbane, Queensland, Australia

e-mail: jysting@icloud.com

Tel.: +61-7-38432541

Fax: +61-7-31631661

\section{J. Y. Ting}

Division of Anaesthesiology and Critical Care, University of Queensland Medical School, Brisbane, Queensland 4067, Australia 